\title{
INITIAL VALUE PROBLEM FOR FRACTIONAL VOLTERRA INTEGRODIFFERENTIAL PSEUDO-PARABOLIC EQUATIONS
}

\author{
Nguyen Duc Phuong ${ }^{1}$, Nguyen Anh Tuan ${ }^{2}$, Devendra Kumar ${ }^{3}$ \\ And NGuYen HuY TuAN ${ }^{4, *}$
}

\begin{abstract}
In this paper, we investigate a initial value problem for the Caputo time-fractional pseudoparabolic equations with fractional Laplace operator of order $0<\nu \leq 1$ and the nonlinear memory source term. For $0<\nu<1$, the problem will be considered on a bounded domain of $\mathbb{R}^{d}$. By some Sobolev embeddings and the properties of the Mittag-Leffler function, we will give some results on the existence and the uniqueness of mild solution for problem (1.1) below. When $\nu=1$, we will introduce some $L^{p}-L^{q}$ estimates, and based on them we derive the global existence of a mild solution in the whole space $\mathbb{R}^{d}$.
\end{abstract}

Mathematics Subject Classification. 26A33, 35R11.

Received June 15, 2020. Accepted February 28, 2021.

\section{INTRODUCTION}

In the last decades, the number of research works on derivatives of non-integer increased sharply. It has been shown that these new types of derivatives are more adequate than the classical integer ones. Fractional derivatives give us a potential instrument for the description of memory hereditary properties of various materials and processes, which are neglected with the classical integer derivative. Fractional derivatives also appear in the theory of control of dynamical systems or fractals. Naturally, the development of Fractional derivatives leads us to the partial differential equations of fractional order. This new type of partial differential equations is being studied more and more by many mathematicians around the world. For studies of the partial differential equation with the fractional derivatives, we refer readers to $[3-5,14,15,18,25-29]$. The following is the statement of the problem.

Keywords and phrases: Fractional partial differential equation, Caputo fractional, well-posedness, pseudo-parabolic equation.

${ }^{1}$ Faculty of Fundamental Science, Industrial University of Ho Chi Minh City, Vietnam.

2 Division of Applied Mathematics, Thu Dau Mot University, Binh Duong Province, Vietnam.

3 Department of Mathematics, University of Rajasthan, Jaipur 302004, Rajasthan, India.

${ }^{4}$ Division of Applied Mathematics, Thu Dau Mot University, Binh Duong Province, Vietnam.

* Corresponding author: nguyenhuytuan@tdmu.edu.vn 
For $\nu \in(0,1], k>0$ and $T$ be a positive constant, various physical phenomena have led us to study the fractional pseudo-parabolic equations with memory term of the form

$$
\begin{cases}{ }_{C} D_{t}^{\alpha}(u-k \Delta u)(x, t)+(-\Delta)^{\nu} u(x, t)=F(u)+\int_{0}^{t} h(t-s, u(s)) \mathrm{d} s, & (x, t) \in \mathcal{D} \times(0, T), \\ u(x, t)=u_{0}(x) & (x, t) \in \mathcal{D} \times\{0\} .\end{cases}
$$

Where $F, h$ are non-linear functions and $u_{0}$ is initial data of the phenomena. The notation ${ }_{C} D_{t}^{\alpha}$ stands for the Caputo time fractional derivative of order $\alpha \in(0,1)$, which can be defined by

$$
{ }_{C} D_{t}^{\alpha} v(t)=\frac{1}{\Gamma(1-\alpha)} \int_{a}^{t} \frac{v^{\prime}(\tau)}{(t-\tau)^{\alpha}} \mathrm{d} \tau
$$

here $v \in H^{1}(a, b)$ and $\Gamma$ is the usual Gamma function.

For $0<\nu<1$, the fractional Laplace operator $(-\Delta)^{\nu}$ is as follow

$$
(\Delta)^{\nu} u(x, t)=\frac{4^{\nu} \Gamma\left(\frac{d}{2}+\nu\right)}{\pi^{\frac{d}{2}}|\Gamma(-\nu)|} \operatorname{P.V} \int_{\mathbb{R}^{d}} \frac{u(x, t)-u(z, t)}{|x-z|^{d+2 \nu}} \mathrm{d} z
$$

this operator approximate the usual Laplacian as $\nu \rightarrow 1$ (see [10]).

In problem (1.1), if we take $\alpha=\nu=1$, we have the partial differential equations which are called pseudoparabolic equations. There are two main reasons why we call this class of equations pseudo-parabolic equations. First, the problems which are well-posed for the parabolic equation are also well-posed for the pseudo-parabolic equations. Second, a solution of the parabolic equation can be approximated by a solution of this type of partial differential equations. The pseudo-parabolic equations have an important role in modeling some physical phenomena such as the unidirectional propagation of nonlinear, dispersive, long waves (see [19]), and the aggregation of the population (see [23]). Our problem is formulated with the fractional operator $(-\Delta)^{\nu}$, which can be regarded as the infinitesimal generators of Lévy stable diffusion processes (see [22]). Let us list some works on the pseudo-parabolic equations with integer order. In [30] Showalter and Ting investigated the equation above

$$
\frac{\partial}{\partial t} u-\eta \Delta \frac{\partial}{\partial t} u=k \Delta u
$$

This equation was generalized by adding the nonlinear source term of the following form

$$
\frac{\partial}{\partial t} u-k \Delta u-\eta \Delta \frac{\partial}{\partial t} u=F(u)
$$

Some common cases for nonlinear source functions can be mentioned as $F(u)=u^{p}, p \geq 1$ (see [7, 32]) and the logarithmic nonlinear terms (see $[11,16]$ ).

However, to the best of our knowledge, the pseudo-parabolic equations with fractional operators like ours have only recently flourished. Some recent works on this topic may be listed as follows. The boundary value problems for a third-order pseudo-parabolic equation with variable coefficients and with the Caputo fractional derivative were studied in [6]. The author presented a priori estimates and based on them, he proved the uniqueness of the solution of these problems and its stability of his problem.

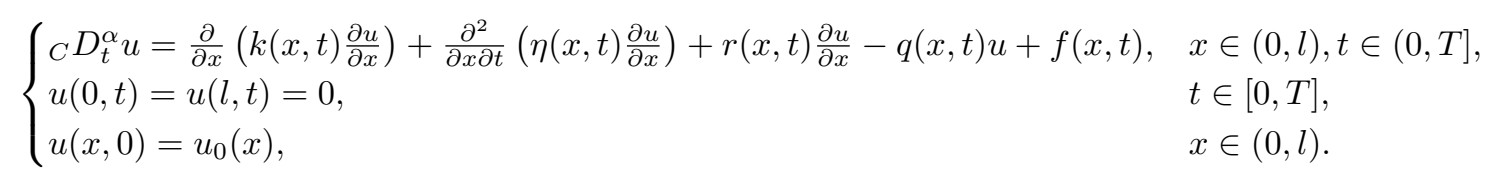


For $\nu \in(0,1)$, in [17], the authors introduced the time-weighted energy method to overcome the weakly dissipative property of the equation and established the global existence and time-decay rates for small-amplitude solutions to the Cauchy problem $\mathrm{m}$ of the fractional pseudo-parabolic equation on the whole space $\mathbb{R}^{d}, d \geq 1$.

We can consider the problem (1.1) to be a generalization of the classical pseudo-parabolic equations. Furthermore, in our model, you will see the presence of $\int_{0}^{t} h(t-z, u(z))$, which can be called the memory term. Although this memory term only makes the calculation techniques a little harder, in terms of practical significance, it has a lot of value in application. For example, in [1], the authors considered memory effects on the dynamics of non-Newtonian fluids and viscoelastic models for the dynamics of turbulence statistics in Newtonian fluids. The comparison of the influence of internal motion and memory effect on energy dissipation was studied by Jaime E. Muñoz Rivera and Luci Harue Fatori in [24].

The above works about this topic are our motivation to study the problem (1.1). Our main goal consists of two parts.

- Part 1: We will prove the global well-posed results for problem (1.1) on a bounded domain $\mathcal{D} \subset \mathbb{R}^{d}$.

- Part 2: By using the $L^{p}-L^{q}$ estimate technique, the global existence and uniqueness of mild solution for problem (1.1) on the whole space $\mathbb{R}^{d}$ will be given.

The outline of this paper is as follows. In Section 2, we introduce some important vector spaces, some properties of the Mittag-Leffler function, and the definition for the mild solutions of (1.1) is also given. In Section 3, we consider the global results for problem (1.1) when $\mathcal{D} \subset \mathbb{R}^{d}$ is a bounded domain with smooth boundary and the source terms are globally Lipschitz in the senses of $L^{2}(\mathcal{D})$. Finally, in the last section, when the domain $\mathcal{D} \equiv \mathbb{R}^{d}$, the global well-posed result will be given for problem (1.1) by using $L^{p}-L^{q}$ estimates method.

\section{Preliminary MATERial}

Sometime in our work, we use the notation $C$ to dedicate a positive constant independent of $u, x, t$.

\subsection{Basic settings}

(see [31]) We first consider the spectral problem for the negative Laplacian on a bounded domain $\mathcal{D} \subset \mathbb{R}^{N}$ as follows

$$
\begin{cases}-\Delta e_{i}(x)=\rho_{i} e_{i}(x), & x \in \mathcal{D} \\ e_{i}(x)=0, & x \in \partial \mathcal{D}\end{cases}
$$

where $0<\rho_{1} \leq \rho_{2} \leq \rho_{3} \leq \cdots \leq \rho_{i} \cdots \nearrow \infty$ and the eigenfunctions's set $\left\{e_{i}\right\}_{i \in \mathbb{N}}$ constitutes an orthonormal basis of $L^{2}(\mathcal{D})$.

We denote the norm on the Banach space $X$ by $\|\cdot\|_{X}$. For $1 \leq p \leq \infty$, the notation $L^{p}(0, T ; X)$ presents the Banach space of measurable functions $v:(0, T) \rightarrow X$, equipped with the norm

$$
\|v\|_{L^{p}(0, T ; X)}=\left(\int_{0}^{T}\|v(t)\|_{X}^{p} \mathrm{~d} t\right)^{\frac{1}{p}}, \quad \text { for } 1 \leq p<\infty,
$$

and

$$
\|v\|_{L^{\infty}(0, T ; X)}=\operatorname{esssup}_{t \in(0, T)}\|v(t)\|_{X} .
$$

Throughout of this work, we set $Z_{0}=L^{p}\left(\mathbb{R}^{d}\right) \cap L^{q}\left(\mathbb{R}^{d}\right)$. The norm of a function $v$ in $Z_{0}$ will be formulated as

$$
\|v\|_{Z_{0}}=\|v\|_{L^{p}\left(\mathbb{R}^{d}\right)}+\|v\|_{L^{q}\left(\mathbb{R}^{d}\right)} .
$$


Let $\mathcal{D} \subset \mathbb{R}^{d}$ and $k$ be a fixed number in $(0,1)$. For any $p \in[1, \infty)$, we can define the fractional Sobolev-type spaces $W^{k, p}(\mathcal{D})$ by (see [10])

$$
W^{k, p}(\mathcal{D}):=\left\{v \in L^{p}(\mathcal{D}) \mid \frac{|v(x)-v(y)|}{|x-y|^{\frac{d+p k}{p}}} \in L^{p}(\mathcal{D} \times \mathcal{D})\right\},
$$

the corresponding norm on $W^{k, p}(\mathcal{D})$ is

$$
\|v\|_{W^{k, p}(\mathcal{D})}=\left(\int_{\mathcal{D}}|v|^{p} d x+\int_{\mathcal{D}} \int_{\mathcal{D}} \frac{|v(x)-v(y)|}{|x-y|^{\frac{d+p k}{p}}} \mathrm{~d} x \mathrm{~d} y\right)^{\frac{1}{p}} .
$$

For $0 \leq m \leq \infty$, the space of all $m$ time derivative continuous functions will be denoted by $C^{m}([0, T] ; X)$. This space is a Banach space with respect to the following norm

$$
\|v\|_{C^{m}([0, T] ; X)}=\sum_{i=1}^{m} \sup _{t \in[0, T]}\left\|v^{(i)}(t)\right\|_{X}<\infty .
$$

For any $\nu>0$, we define the Hilbert scale space

$$
\mathcal{H}^{\nu}(\mathcal{D})=\left\{v \in L^{2}(\mathcal{D}):\|v\|_{\mathcal{H}^{\nu}(\mathcal{D})}^{2}=\sum_{i=1}^{\infty} \rho_{i}^{2 \nu}\left\langle v(\cdot), e_{i}(\cdot)\right\rangle^{2}<\infty\right\},
$$

and the Hilbert space $\mathcal{H}^{-\nu}(\mathcal{D})$ with the norm

$$
\|v\|_{\mathcal{H}^{-\nu}(\mathcal{D})}=\left(\sum_{i=1}^{\infty} \rho_{i}^{-2 \nu}\left\langle v(\cdot), e_{i}(\cdot)\right\rangle_{*}^{2}\right)^{\frac{1}{2}}
$$

which is the duality space of $\mathcal{H}^{\nu}(\mathcal{D})$. The notation $\langle\cdot, \cdot\rangle_{*}$ denotes the dual inner product between $\mathcal{H}^{-\nu}(\mathcal{D})$ and $\mathcal{H}^{\nu}(\mathcal{D})$. We note that

$$
\langle u, v\rangle_{*}=\langle u, v\rangle, \quad \text { for } u \in L^{2}(\mathcal{D}), v \in \mathcal{H}^{\nu}(\mathcal{D}) .
$$

\subsection{Mittag-Leffler function}

To present the formula of the mild solution to problem (1.1), we need some important properties of the Mittag-Leffler function. First, let us introduce the definition of this function. Let $\alpha>0$, and $\beta \in \mathbb{R}$ are arbitrary constants, $\Gamma$ is the usual Gamma function. The Mittag-Leffler function can be defined by (see [13])

$$
E_{\alpha, \beta}(z)=\sum_{i=1}^{\infty} \frac{z^{i}}{\Gamma(\alpha i+\beta)}, \quad z \in \mathbb{C} .
$$

Lemma 2.1. (see [13]) For $0<\alpha_{1}<\alpha_{2}<1$ and $\alpha \in\left[\alpha_{1}, \alpha_{2}\right]$, there exist the positive constants $m_{\alpha}$ and $M_{\alpha}$, depending only on $\alpha$ such that

(i) $E_{\alpha, 1}(-z)>0$, for $z>0$,

(ii) $\frac{m_{\alpha}}{1+z} \leq E_{\alpha, \beta}(-z) \leq \frac{M_{\alpha}}{1+z}$, for $\beta \in \mathbb{R}, z>0$ 
Definition 2.2. Let $z$ be a complex number. We define the Wright function as

$$
\mathcal{W}_{\alpha}(z):=\sum_{n=0}^{\infty} \frac{z^{n}}{n ! \Gamma(1-(\alpha(n+1)))}, \quad 0<\alpha<1 .
$$

Proposition 2.3. (see [21]) Let $0<\alpha<1$. Then, the following properties hold

(i) for $z \geq 0$, we have $\mathcal{W}_{\alpha}(z) \geq 0$,

(ii) for $\delta>-1$, we have $\int_{0}^{\infty} z^{\delta} \mathcal{W}_{\alpha}(z) \mathrm{d} z=\frac{\Gamma(\delta+1)}{\Gamma(\delta \alpha+1)}$.

Lemma 2.4. Let $\mathcal{M}$ be the infinitesimal generator of an analytic semigroup $\left\{e^{-\mathcal{M} t}\right\}_{t \geq 0}$. The following equalities hold

$$
E_{\alpha, 1}\left(\mathcal{M} t^{\alpha}\right)=\int_{0}^{\infty} \mathcal{W}_{\alpha}(r) e^{-\mathcal{M} r t^{\alpha}} \mathrm{d} r
$$

and

$$
E_{\alpha, \alpha}\left(\mathcal{M} t^{\alpha}\right)=\int_{0}^{\infty} \alpha r \mathcal{W}_{\alpha}(r) e^{-\mathcal{M} r t^{\alpha}} \mathrm{d} r
$$

\subsection{The mild solution formula}

Definition 2.5. A function $u$ is called the mild solution of problem (1.1) if $u$ belongs to $C\left([0, T] ; L^{p}(\mathcal{D})\right), p \geq 1$ and satisfies the following integral equation

$$
\begin{aligned}
u(t)= & E_{\alpha, 1}\left[(-\Delta)^{\nu}(k \Delta-I)^{-1} t^{\alpha}\right] u_{0} \\
& +(I-k \Delta)^{-1} \int_{0}^{t}(t-m)^{\alpha-1} E_{\alpha, \alpha}\left[(-\Delta)^{\nu}(k \Delta-I)^{-1}(t-m)^{\alpha-1}\right] F(u(m)) \mathrm{d} m \\
& +(I-k \Delta)^{-1} \int_{0}^{t} \int_{0}^{m}(t-m)^{\alpha-1} E_{\alpha, \alpha}\left[(-\Delta)^{\nu}(k \Delta-I)^{-1}(t-m)^{\alpha-1}\right] h(t-s, u(s)) \mathrm{d} s \mathrm{~d} m .
\end{aligned}
$$

Remark 2.6. In view of the above definition and the expansion $v(x)=\sum_{i=1}^{\infty}\left\langle v, e_{i}\right\rangle e_{i}(x)$, on a bounded domain of $\mathbb{R}^{d}$, the operator $E_{\alpha, \beta}\left[(-\Delta)^{\nu}(k \Delta-I)^{-1} t^{\alpha}\right]$ can be expressed as

$$
E_{\alpha, \beta}\left[(-\Delta)^{\nu}(k \Delta-I)^{-1} t^{\alpha}\right] v(x)=\sum_{i=1}^{\infty} E_{\alpha, \beta}\left(\frac{-\rho_{i}^{\nu} t^{\alpha}}{1+k \rho_{i}}\right)\left\langle v, e_{i}\right\rangle e_{i}(x) .
$$

Remark 2.7. If $u_{0}(x)$ is positive all everywhere on $\mathcal{D}$ and the continuous function $F, h$ are nonnegative, then mild solution of problem (1.1) is positive.

Remark 2.8. In [20], we see that $\mathcal{M}=-\Delta(I-k \Delta)^{-1}$ is the infinitesimal generator of an analytic semigroup $\left\{e^{-\mathcal{M} t}\right\}_{t \geq 0}$. This remark will be needed in the last section of this paper.

\subsection{The globally Lipschitz functions}

In the rest of our work, we consider the assumptions below for the source functions $F, h$ 


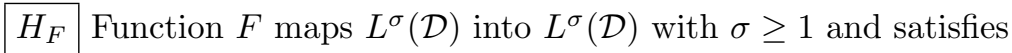

$\left\{\begin{array}{l}\left\|F\left(v_{1}\right)-F\left(v_{2}\right)\right\|_{L^{\sigma}(\mathcal{D})} \leq C_{F}\left\|v_{1}-v_{2}\right\|_{L^{\sigma}(\mathcal{D})}, \\ F(0)=0,\end{array}\right.$

here, $C_{F}$ is positive constants independent of $v_{1}, v_{2}$.

$H_{h}$ Function $h$ maps $\mathbb{R}^{+} \times L^{\sigma}(\mathcal{D})$ into $\mathbb{R}^{+} \times L^{\sigma}(\mathcal{D})$ with $\sigma \geq 1$ and satisfies

$\left\{\begin{array}{l}\left\|h\left(t, v_{1}\right)-h\left(t, v_{2}\right)\right\|_{L^{\sigma}(\mathcal{D})} \leq C_{h} \Phi(t)\left\|v_{1}-v_{2}\right\|_{L^{\sigma}(\mathcal{D})}, \\ h(t, 0)=0, \forall t>0\end{array}\right.$

here, $C_{h}$ is positive constant independent of $v_{1}, v_{2}$ and $\Phi$ satisfies

$$
|\Phi(t)| \leq t^{-\beta}, t>0, \beta \in(0,1) .
$$

\section{Global Result with Bounded domain}

\subsection{Theory result}

Throughout this section, we study the existence and the uniqueness for the mild solution of problem (1.1) under the assumption that $\mathcal{D} \subset \mathbb{R}^{d}$ is a bounded domain with smooth boundary $\partial \mathcal{D}$ and the Dirichlet boundary condition

$$
u(x, t)=0,(x, t) \in \partial \mathcal{D} \times(0, T)
$$

is satisfied. In addition, we suppose that the source terms $F, h$ satisfy $(2.15)$ and (2.16) with $\sigma=2$, respectively. For optimizing our results, we consider a weighted space denoted by $C_{\delta, \mu}(I ; X)$, which is the space of all functions $v \in C(I ; X)$ such that

$$
\|v\|_{C_{\delta, \mu}(I ; X)}:=\sup _{t \in I} t^{\delta} e^{-\mu t}\|v(\cdot, t)\|_{X}<\infty .
$$

We first introduce some useful lemmas before presenting the main results of this section.

Lemma 3.1. (see [2, 12])Let $\mathcal{D} \subset \mathbb{R}^{d}, 0 \leq m \leq k<\infty, \leq p<\infty$. Suppose that $(k-m) p<d$. Then, we have

- $W^{k, p}(\mathcal{D}) \hookrightarrow W^{m, q}(\mathcal{D}), \quad$ for $\quad 1 \leq q<\frac{p d}{d-(k-m) p}$,

- $\mathcal{H}^{\nu}(\mathcal{D}) \hookrightarrow W^{\nu, 2}, \quad$ for $\quad \nu>0$,

- $L^{p}\left(\mathbb{R}^{d}\right) \hookrightarrow \mathcal{H}^{\nu}(\mathcal{D}), \quad$ for $\quad-\frac{d}{2}<\nu \leq 0, \quad p \geq \frac{2 d}{d-2 \nu}$,

- $\mathcal{H}^{\nu}(\mathcal{D}) \hookrightarrow L^{p}\left(\mathbb{R}^{d}\right), \quad$ for $\quad 0 \leq \nu<\frac{d}{2}, \quad p \leq \frac{2 d}{d-2 \nu}$.

Lemma 3.2. (see [9]) Let $a, b>-1$ such that $a+b>-1$. Then, for $\delta>0$ and $t \in[0, T]$, we have

$$
\lim _{\mu \rightarrow \infty}\left(\sup _{t \in[0, T]} t^{\delta} \int_{0}^{1} z^{a}(1-z)^{b} e^{-\mu t(1-z)} \mathrm{d} z\right)=0 .
$$


Theorem 3.3. Let $\alpha, \delta \in(0,1), \nu \in(0,1]$, and $u_{0} \in \mathcal{H}^{\nu}(\mathcal{D})$. Assume that $F, h$ satisfy (2.15) and $(2.16)$, respectively and $\delta+\max \{1-\alpha, \beta\}<1$. Then, for $0<\kappa \leq \nu, 1 \leq p \leq \frac{2 d}{d+2 \kappa-2 \nu}$ such that $\kappa p<d$ and $\frac{d p}{d-\kappa p} \geq 2$, problem (1.1) has a unique mild solution $u \in C_{\delta, \mu}\left([0, T] ; W^{\kappa, p}(\mathcal{D})\right)$ for some $\mu>0$.

Proof. We begin the proof by defining the operator $\mathcal{J}$ from $C_{\delta, \mu}\left([0, T] ; W^{\kappa, p}(\mathcal{D})\right)$ to $C_{\delta, \mu}\left([0, T] ; W^{\kappa, p}(\mathcal{D})\right)$ by

$$
\begin{aligned}
\mathcal{J} v(t)= & E_{\alpha, 1}\left[(-\Delta)^{\nu}(k \Delta-I)^{-1} t^{\alpha}\right] u_{0} \\
& +(I-k \Delta)^{-1} \int_{0}^{t}(t-m)^{\alpha-1} E_{\alpha, \alpha}\left[(-\Delta)^{\nu}(k \Delta-I)^{-1}(t-m)^{\alpha-1}\right] F(v(m)) \mathrm{d} m \\
& +(I-k \Delta)^{-1} \int_{0}^{t}(t-m)^{\alpha-1} E_{\alpha, \alpha}\left[(-\Delta)^{\nu}(k \Delta-I)^{-1}(t-m)^{\alpha-1}\right] \int_{0}^{m} h(t-s, v(s)) \mathrm{d} s \mathrm{~d} m \\
= & : J_{0}+J_{F}(v)+J_{h}(v) .
\end{aligned}
$$

Our main idea is to apply the Banach principle argument to show that $\mathcal{J}$ has a unique fixed point on $C_{\delta, \mu}\left([0, T] ; W^{\kappa, p}(\mathcal{D})\right)$. Firstly, for $u_{0} \in \mathcal{H}^{\nu}(\mathcal{D})$, thanks to Lemma 2.1, we find that

$$
\begin{aligned}
t^{2 \delta} e^{-2 \mu t}\left\|E_{\alpha, 1}\left[(-\Delta)^{\nu}(k \Delta-I)^{-1} t^{\alpha}\right] u_{0}\right\|_{\mathcal{H}^{\nu}(\mathcal{D})}^{2} & =T^{2 \delta} \sum_{i=1}^{\infty}\left\langle u_{0}, e_{i}\right\rangle^{2} E_{\alpha, 1}\left(-\frac{\rho_{i}^{\nu} t^{\alpha}}{1+k \rho_{i}}\right) \rho_{i}^{2 \nu} \\
& \leq T^{2 \delta} \sum_{i=1}^{\infty}\left(\frac{M_{\alpha}}{1+\frac{\rho_{i}^{\nu} t^{\alpha}}{1+k \rho_{i}}}\right)^{2}\left\langle u_{0}, e_{i}\right\rangle^{2} \rho_{i}^{2 \nu} \\
& \leq\left(T^{\delta} M_{\alpha}\right)^{2} \sum_{i=1}^{\infty}\left\langle u_{0}, e_{i}\right\rangle^{2} \rho_{i}^{2 \nu}<\infty .
\end{aligned}
$$

By using Lemma 3.1 with the conditions $\kappa \leq \nu$ and $1 \leq p \leq \frac{2 d}{d+2 \kappa-2 \nu}$, we have

$$
\mathcal{H}^{\nu}(\mathcal{D}) \hookrightarrow W^{\nu, 2}(\mathcal{D}) \hookrightarrow W^{\kappa, p}(\mathcal{D})
$$

From the two results above, we obtain

$$
\left\|E_{\alpha, 1}\left[(-\Delta)^{\nu}(k \Delta-I)^{-1} t^{\alpha}\right] u_{0}\right\|_{C_{\delta, \mu}\left([0, T] ; W^{\kappa, p}(\mathcal{D})\right)} \leq T^{\delta} M_{\alpha}\left\|u_{0}\right\|_{\mathcal{H}^{\nu}(\mathcal{D})}
$$

It follows immediately from the property of the solution representation operator and the above result that $J_{0} \in C_{\delta, \mu}\left([0, T] ; W^{\kappa, p}(\mathcal{D})\right)$. Now, we will show that $J$ is a contraction mapping on $C_{\delta, \mu}\left([0, T] ; W^{\kappa, p}(\mathcal{D})\right)$. Let $v_{1}, v_{2}$ belong to $C_{\delta, \mu}\left([0, T] ; W^{\kappa, p}(\mathcal{D})\right)$, we derive two important estimates as follows.

Estimate 1. Parseval's identity gives us

$$
\begin{aligned}
& \left\|J_{F}\left(v_{1}(t)\right)-J_{F}\left(v_{2}(t)\right)\right\|_{\mathcal{H}^{\nu}(\mathcal{D})} \\
& \leq \int_{0}^{t}(t-m)^{\alpha-1}\left(\sum_{i=1}^{\infty}\left(\frac{\rho_{i}^{\nu}}{1+k \rho_{i}}\right)^{2} E_{\alpha, \alpha}^{2}\left(-\frac{\rho_{i}^{\nu}(t-m)^{\alpha-1}}{1+k \rho_{i}}\right)\left\langle F\left(v_{1}(m)\right)-F\left(v_{2}(m)\right), e_{i}\right\rangle^{2}\right)^{\frac{1}{2}} \mathrm{~d} m .
\end{aligned}
$$

Using Lemma 2.1, we obtain

$$
\left\|J_{F}\left(v_{1}(t)\right)-J_{F}\left(v_{2}(t)\right)\right\|_{\mathcal{H}^{\nu}(\mathcal{D})} \leq \frac{M_{\alpha}}{k} \int_{0}^{t}(t-m)^{\alpha-1}\left\|F\left(v_{1}(m)\right)-F\left(v_{2}(m)\right)\right\|_{\mathcal{H}^{\nu-1}(\mathcal{D})} \mathrm{d} m
$$




$$
\begin{aligned}
& \leq \frac{M_{\alpha} C_{\nu}}{k} \int_{0}^{t}(t-m)^{\alpha-1}\left\|F\left(v_{1}(m)\right)-F\left(v_{2}(m)\right)\right\|_{L^{2}(\mathcal{D})} \mathrm{d} m \\
& \leq \frac{C_{F} M_{\alpha} C_{\nu}}{k} \int_{0}^{t}(t-m)^{\alpha-1}\left\|v_{1}(m)-v_{2}(m)\right\|_{L^{2}(\mathcal{D})} \mathrm{d} m,
\end{aligned}
$$

here, we have used the fact that $\nu \in(0,1)$. We also notice that the Sobolev embedding $W_{\kappa, p}(\mathcal{D}) \hookrightarrow L^{2}(\mathcal{D})$ holds for $\kappa \geq \frac{2 d-d p}{2 p}$. It gives us the following result

$$
\begin{aligned}
& t^{\delta} e^{-\mu t}\left\|J_{F}\left(v_{1}(t)\right)-J_{F}\left(v_{2}(t)\right)\right\|_{\mathcal{H}^{\nu}(\mathcal{D})} \\
& \leq \frac{C_{F} M_{\alpha} C_{\nu}}{k}\left(t^{\delta} \int_{0}^{t}(t-m)^{\alpha-1} m^{-\delta} e^{-\mu(t-m)} \mathrm{d} m\right)\left\|v_{1}-v_{2}\right\|_{C_{\delta, \mu}\left([0, T] ; W^{\kappa, p}(\mathcal{D})\right)} \\
& =\frac{C_{F} M_{\alpha} C_{\nu}}{k}\left(t^{\alpha} \int_{0}^{1}(1-m)^{\alpha-1} m^{-\delta} e^{-\mu t(1-m)} \mathrm{d} m\right)\left\|v_{1}-v_{2}\right\|_{C_{\delta, \mu}\left([0, T] ; W^{\kappa, p}(\mathcal{D})\right)} .
\end{aligned}
$$

Estimate 2. Similarly, we have

$$
\begin{aligned}
& t^{\delta} e^{-\mu t}\left\|J_{h}\left(v_{1}\right)-J_{h}\left(v_{2}\right)\right\|_{\mathcal{H}^{\nu}(\mathcal{D})} \\
& \leq \frac{C_{h} M_{\alpha} C_{\nu}}{C_{\Phi}^{-1} k}\left[t^{\delta} \int_{0}^{t} \frac{(t-m)^{\alpha-1}}{m^{\delta} e^{\mu(t-m)}}\left(m^{\delta} \int_{0}^{m} \frac{(m-s)^{-\beta}}{s^{\delta} e^{\mu(m-s)}} \mathrm{d} s\right) \mathrm{d} m\right]\left\|v_{1}-v_{2}\right\|_{C_{\delta, \mu}\left([0, T] ; W^{\kappa, p}(\mathcal{D})\right)} \\
& =\frac{C_{h} M_{\alpha} C_{\nu}}{C_{\Phi}^{-1} k}\left[t^{\alpha} \int_{0}^{1} \frac{(1-m)^{\alpha-1}}{m^{\delta} e^{\mu t(1-m)}}\left(m^{1-\beta} \int_{0}^{1} \frac{(1-s)^{-\beta}}{s^{\delta} e^{\mu s(1-s)}} \mathrm{d} s\right) \mathrm{d} m\right]\left\|v_{1}-v_{2}\right\|_{C_{\delta, \mu}\left([0, T] ; W^{\kappa, p}(\mathcal{D})\right)} .
\end{aligned}
$$

Applying Lemma 3.2, we get the following limits

$$
\left\{\begin{array}{l}
\lim _{\mu \rightarrow \infty}\left(t^{\alpha} \int_{0}^{1}(1-m)^{\alpha-1} m^{-\delta} e^{-\mu t(1-m)} \mathrm{d} m\right)=0 \\
\lim _{\mu \rightarrow \infty}\left(t^{1-\beta} \int_{0}^{1}(1-m)^{-\beta} m^{-\delta} e^{-\mu t(1-m)} \mathrm{d} m\right)=0
\end{array}\right.
$$

Combining the above results, we can find a sufficiently large $\mu$ such that

$$
\left\|\mathcal{J} v_{1}-\mathcal{J} v_{2}\right\|_{C_{\delta, \mu}\left([0, T] ; W^{\kappa, p}(\mathcal{D})\right)} \leq \mathcal{L}\left\|v_{1}-v_{2}\right\|_{C_{\delta, \mu}\left([0, T] ; W^{\kappa, p}(\mathcal{D})\right)},
$$

where $\mathcal{L}$ is a positive constant which is less than 1 . It's not difficult to check that $\mathcal{J}$ is continous on $[0, T]$ and $C_{\delta, \mu}\left([0, T] ; W^{\kappa, p}(\mathcal{D})\right)$ is a complete metric space. Then, $\mathcal{J}$ is an invariant contraction on $C_{\delta, \mu}\left([0, T] ; W^{\kappa, p}(\mathcal{D})\right)$. Hence, we can conclude that problem (1.1) has a unique mild solution in $C_{\delta, \mu}\left([0, T] ; W^{\kappa, p}(\mathcal{D})\right)$.

\subsection{Application}

We apply our theory result to the following initial value problem

$$
\begin{cases}{ }_{C} D_{t}^{1 / 2}(u-k \Delta u)=\Delta u+\lambda_{1} u+\int_{0}^{t} \lambda_{2}(t-z)^{-0,45} u(z) \mathrm{d} z, & \text { in } \mathcal{B} \times(0, T), \\ u=0, & \text { in } \partial \mathcal{B} \times(0, T) \\ u=0 & \text { in } \mathcal{B} \times\{0\}\end{cases}
$$


here $d=3, \mathcal{B}=\mathcal{B}(0, \mathcal{Z})$ is a three-dimension open ball centered at the origin with radius $\mathcal{Z}>0$, and $\lambda_{1}, \lambda_{2}$ are positive constants. It's obvious that $F(u)=\lambda_{1} u$ and $h(t, u)=\lambda_{2} t^{-0,45} u$ satisfy $(2.15)$ and (2.16), respectively. Then, Theorem 3.3 implies the unique existence of the mild solution of this problem in the space $C_{0,45, \mu_{0}}\left([0, T] ; H^{1}(\mathcal{B})\right)$, for some large positive $\mu_{0}$.

\section{Global existence And uniqueness Result By $L^{p}-L^{q}$ EStimates}

\subsection{Theory result}

In this section, problem (1.1) will be considered in the case $\nu=1$, and the domain $\mathcal{D}$ is identified with the whole space $\mathbb{R}^{d}$. The source terms $F, h$ satisfy (2.15) and (2.16), respectively. For purpose of proving the global existence for the mild solution of the problem (1.1), we introduce the following important lemma.

Lemma 4.1. (see [8]) Let $1 \leq p \leq q$ and assume that $v \in Z_{0}$. Then, there exist a constant $\widetilde{C}>0$ such that

$$
\begin{aligned}
& \left\|e^{-\mathcal{M} t} w\right\|_{L^{q}\left(\mathbb{R}^{d}\right)} \leq \widetilde{C}\left[(1+t)^{\frac{d}{2}\left(\frac{1}{q}-\frac{1}{p}\right)}\|w\|_{L^{p}\left(\mathbb{R}^{d}\right)}+e^{-t}\|w\|_{L^{q}\left(\mathbb{R}^{d}\right)}\right] \\
& \left\|(I-k \Delta)^{-1} w\right\|_{L^{q}\left(\mathbb{R}^{d}\right)} \leq \widetilde{C}\|w\|_{L^{q}\left(\mathbb{R}^{d}\right)} .
\end{aligned}
$$

Theorem 4.2. Let $1 \leq p \leq q$ such that

$$
\frac{1}{p}-\frac{1}{q}<\frac{2}{d}
$$

and

$$
\frac{\alpha d(q-p)}{2 p q}<\delta<\max \left\{\alpha+\frac{\alpha d(p-q)}{2 p q}, 1-\beta\right\} .
$$

Assume that $u_{0} \in Z_{0}$ and $F, h$ satisfy (2.15), (2.16), respectively. Then, for any $T \in(0, \infty)$, problem (1.1) has a unique mild solution in $C_{\delta, \mu}\left((0, T] ; Z_{0}\right)$.

Proof. Before working on the main idea, we state some useful estimates. First, it's easily seen that the inequality $e^{-r} \leq c_{\epsilon} r^{-\epsilon}$ holds for any $r>0$ with some $\epsilon \in(0,1)$. This fact and Lemma 4.1 lead us to the following

$$
\begin{aligned}
\int_{0}^{\infty} & \mathcal{W}_{\alpha}(r)\left\|e^{-\mathcal{M} r t^{\alpha}} u_{0}\right\|_{L^{q}\left(\mathbb{R}^{d}\right)} \mathrm{d} r \\
\leq & \widetilde{C} \int_{0}^{\infty} \mathcal{W}_{\alpha}(r)\left[\left(1+r t^{\alpha}\right)^{\frac{d}{2}\left(\frac{1}{q}-\frac{1}{p}\right)}\left\|u_{0}\right\|_{L^{p}\left(\mathbb{R}^{d}\right)}+e^{-r t^{\alpha}}\left\|u_{0}\right\|_{L^{q}\left(\mathbb{R}^{d}\right)}\right] \mathrm{d} r \\
\leq & \widetilde{C} t^{\frac{\alpha d}{2}\left(\frac{1}{q}-\frac{1}{p}\right)}\left\|u_{0}\right\|_{L^{p}\left(\mathbb{R}^{d}\right)} \int_{0}^{\infty} \mathcal{W}_{\alpha}(r) r^{\frac{d}{2}\left(\frac{1}{q}-\frac{1}{p}\right)} \mathrm{d} r+\widetilde{C} c_{\epsilon} t^{-\alpha \epsilon}\left\|u_{0}\right\|_{L^{q}\left(\mathbb{R}^{d}\right)} \int_{0}^{\infty} \mathcal{W}_{\alpha}(r) r^{-\epsilon} \mathrm{d} r \\
= & \frac{\widetilde{C} \Gamma\left(1+\frac{d}{2}\left(\frac{1}{q}-\frac{1}{p}\right)\right)}{\Gamma\left(1+\frac{\alpha d}{2}\left(\frac{1}{q}-\frac{1}{p}\right)\right)} t^{\frac{\alpha d}{2}\left(\frac{1}{q}-\frac{1}{p}\right)}\left\|u_{0}\right\|_{L^{p}\left(\mathbb{R}^{d}\right)}+\frac{\widetilde{C} c_{\epsilon} \Gamma(1-\epsilon)}{\Gamma(1-\alpha \epsilon)} t^{-\alpha \epsilon}\left\|u_{0}\right\|_{L^{q}\left(\mathbb{R}^{d}\right)} .
\end{aligned}
$$

By choosing $\epsilon=\frac{d}{2}\left(\frac{1}{p}-\frac{1}{q}\right) \in(0,1)$, the latter inequality becomes

$$
\int_{0}^{\infty} \mathcal{W}_{\alpha}(r)\left\|e^{-\mathcal{M} r t^{\alpha}} u_{0}\right\|_{L^{q}\left(\mathbb{R}^{d}\right)} \mathrm{d} r \leq \frac{\widetilde{C} \Gamma\left(1+\frac{d}{2}\left(\frac{1}{q}-\frac{1}{p}\right)\right)}{\Gamma\left(1+\frac{\alpha d}{2}\left(\frac{1}{q}-\frac{1}{p}\right)\right)} t^{\frac{\alpha d}{2}\left(\frac{1}{q}-\frac{1}{p}\right)}\left(\left\|u_{0}\right\|_{L^{p}\left(\mathbb{R}^{d}\right)}+c_{\epsilon}\left\|u_{0}\right\|_{L^{q}\left(\mathbb{R}^{d}\right)}\right)
$$




$$
\leq C t^{\frac{\alpha d}{2}\left(\frac{1}{q}-\frac{1}{p}\right)}\left(\left\|u_{0}\right\|_{L^{p}\left(\mathbb{R}^{d}\right)}+\left\|u_{0}\right\|_{L^{q}\left(\mathbb{R}^{d}\right)}\right) .
$$

Thanks to Lemma 2.4 and the above, we obtain

$$
\begin{aligned}
\left\|E_{\alpha, 1}\left[\Delta(I-k \Delta)^{-1} t^{\alpha}\right] u_{0}\right\|_{L^{q}\left(\mathbb{R}^{d}\right)} & \leq \int_{0}^{\infty} \mathcal{W}_{\alpha}(r)\left\|e^{-\mathcal{M} r t^{\alpha}} u_{0}\right\|_{L^{q}\left(\mathbb{R}^{d}\right)} \mathrm{d} r \\
& \leq C t^{\frac{\alpha d}{2}\left(\frac{1}{q}-\frac{1}{p}\right)}\left(\left\|u_{0}\right\|_{L^{p}\left(\mathbb{R}^{d}\right)}+\left\|u_{0}\right\|_{L^{q}\left(\mathbb{R}^{d}\right)}\right) .
\end{aligned}
$$

In Lemma 4.1, if we take $p=q$, the following holds

$$
\begin{aligned}
\left\|E_{\alpha, 1}\left[\Delta(I-k \Delta)^{-1} t^{\alpha}\right] u_{0}\right\|_{L^{p}\left(\mathbb{R}^{d}\right)} & \leq \widetilde{C} \int_{0}^{\infty} \mathcal{W}_{\alpha}(r)\left[\left\|u_{0}\right\|_{L^{p}\left(\mathbb{R}^{d}\right)}+e^{-r t^{\alpha}}\left\|u_{0}\right\|_{L^{p}\left(\mathbb{R}^{d}\right)}\right] \mathrm{d} r \\
& \leq 2 \widetilde{C}\left\|u_{0}\right\|_{L^{p}\left(\mathbb{R}^{d}\right)} \int_{0}^{\infty} \mathcal{W}_{\alpha}(r) \mathrm{d} r=2 \widetilde{C}\left\|u_{0}\right\|_{L^{p}\left(\mathbb{R}^{d}\right)},
\end{aligned}
$$

here we notice that $e^{-r t^{\alpha}} \leq 1$. Next, for a function $w \in Z_{0}$, by the same method as the above estimates, we find that

$$
\begin{aligned}
& \int_{0}^{\infty} \alpha r \mathcal{W}_{\alpha}(r)\left\|e^{-\mathcal{M} r t^{\alpha}} w\right\|_{L^{q}\left(\mathbb{R}^{d}\right)} \mathrm{d} r \\
& \leq \widetilde{C} \alpha t^{\frac{\alpha d}{2}\left(\frac{1}{q}-\frac{1}{p}\right)}\|w\|_{L^{p}\left(\mathbb{R}^{d}\right)} \int_{0}^{\infty} \mathcal{W}_{\alpha}(r) r^{1+\frac{d}{2}\left(\frac{1}{q}-\frac{1}{p}\right)} \mathrm{d} r+\widetilde{C} c_{\epsilon} \alpha t^{-\alpha \epsilon}\|w\|_{L^{q}\left(\mathbb{R}^{d}\right)} \int_{0}^{\infty} \mathcal{W}_{\alpha}(r) r^{1-\epsilon} \mathrm{d} r \\
& =\frac{\widetilde{C} \alpha \Gamma\left(2+\frac{d}{2}\left(\frac{1}{q}-\frac{1}{p}\right)\right)}{\Gamma\left(1+\alpha+\frac{\alpha d}{2}\left(\frac{1}{q}-\frac{1}{p}\right)\right)} t^{\frac{\alpha d}{2}\left(\frac{1}{q}-\frac{1}{p}\right)}\|w\|_{L^{p}\left(\mathbb{R}^{d}\right)}+\frac{\widetilde{C} c_{\epsilon} \alpha \Gamma(2-\epsilon)}{\Gamma(1+\alpha-\alpha \epsilon)} t^{-\alpha \epsilon}\|w\|_{L^{q}\left(\mathbb{R}^{d}\right)} \\
& \leq \frac{\widetilde{C} \alpha \Gamma\left(2+\frac{d}{2}\left(\frac{1}{q}-\frac{1}{p}\right)\right)}{\Gamma\left(1+\alpha+\frac{\alpha d}{2}\left(\frac{1}{q}-\frac{1}{p}\right)\right)} \max \left\{1, c_{\epsilon}\right\} t^{\frac{\alpha d}{2}\left(\frac{1}{q}-\frac{1}{p}\right)}\left(\|w\|_{L^{p}\left(\mathbb{R}^{d}\right)}+\|w\|_{L^{q}\left(\mathbb{R}^{d}\right)}\right)
\end{aligned}
$$

It follows immediately that

$$
\begin{aligned}
\left\|E_{\alpha, \alpha}\left[\Delta(I-k \Delta)^{-1} t^{\alpha}\right] w\right\|_{L^{q}\left(\mathbb{R}^{d}\right)} & \leq \int_{0}^{\infty} \alpha r \mathcal{W}_{\alpha}(r)\left\|e^{-\mathcal{M} r t^{\alpha}} w\right\|_{L^{q}\left(\mathbb{R}^{d}\right)} \mathrm{d} r \\
& \leq C t^{\frac{\alpha d}{2}\left(\frac{1}{q}-\frac{1}{p}\right)}\left(\|w\|_{L^{p}\left(\mathbb{R}^{d}\right)}+\|w\|_{L^{q}\left(\mathbb{R}^{d}\right)}\right) .
\end{aligned}
$$

Similar arguments can be applied to the $L^{p}\left(\mathbb{R}^{d}\right)$ norm of $w$. Then, we get

$$
\begin{aligned}
\left\|E_{\alpha, \alpha}\left[\Delta(I-k \Delta)^{-1} t^{\alpha}\right] w\right\|_{L^{p}\left(\mathbb{R}^{d}\right)} & \leq \widetilde{C} \alpha \int_{0}^{\infty} r \mathcal{W}_{\alpha}(r)\left[\|w\|_{L^{p}\left(\mathbb{R}^{d}\right)}+e^{-r t^{\alpha}}\|w\|_{L^{p}\left(\mathbb{R}^{d}\right)}\right] \mathrm{d} r \\
& \leq 2 \widetilde{C} \alpha\|w\|_{L^{p}\left(\mathbb{R}^{d}\right)} \int_{0}^{\infty} r \mathcal{W}_{\alpha}(r) \mathrm{d} r=2 \widetilde{C} \alpha \frac{\Gamma(2)}{\Gamma(1+\alpha)}\|w\|_{L^{p}\left(\mathbb{R}^{d}\right)} .
\end{aligned}
$$

We are now in a position to present the main point of this proof. To this end, we define a mapping $\mathscr{K}$ from $C_{\delta, \mu}\left((0, T] ; Z_{0}\right)$ into $C_{\delta, \mu}\left((0, T] ; Z_{0}\right)$ by

$$
\mathscr{K} v(t)=E_{\alpha, 1}\left[\Delta(I-k \Delta)^{-1} t^{\alpha}\right] u_{0}
$$


INITIAL VALUE PROBLEM FOR FRACTIONAL VOLTERRA INTEGRODIFFERENTIAL PSEUDO-PARABOLIC EQUATIONS 11

$$
\begin{aligned}
& +(I-k \Delta)^{-1} \int_{0}^{t}(t-m)^{\alpha-1} E_{\alpha, \alpha}\left[\Delta(I-k \Delta)^{-1}(t-m)^{\alpha-1}\right] F(v(m)) \mathrm{d} m \\
& +(I-k \Delta)^{-1} \int_{0}^{t}(t-m)^{\alpha-1} E_{\alpha, \alpha}\left[\Delta(I-k \Delta)^{-1}(t-m)^{\alpha-1}\right] \int_{0}^{m} h(t-s, v(s)) \mathrm{d} s \mathrm{~d} m .
\end{aligned}
$$

From (4.5), (4.6) and the assumption that $u_{0} \in Z_{0}$, we have

$$
\begin{aligned}
& t^{\delta} e^{-\mu t}\left\|E_{\alpha, 1}\left[\Delta(I-k \Delta)^{-1} t^{\alpha}\right] u_{0}\right\|_{Z_{0}} \\
& \leq e^{-\mu t}\left(C_{0} t^{\delta+\frac{\alpha d}{2}\left(\frac{1}{q}-\frac{1}{p}\right)}+2 C t^{\delta}\right)\left\|u_{0}\right\|_{L^{p}\left(\mathbb{R}^{d}\right)}+C_{0} e^{-\mu t} t^{\delta+\frac{\alpha d}{2}\left(\frac{1}{q}-\frac{1}{p}\right)}\left\|u_{0}\right\|_{L^{q}\left(\mathbb{R}^{d}\right)} \\
& \leq\left(C_{0} T^{\delta+\frac{\alpha d}{2}\left(\frac{1}{q}-\frac{1}{p}\right)}+2 C T^{\delta}\right)\left\|u_{0}\right\|_{L^{p}\left(\mathbb{R}^{d}\right)}+C_{0} T^{\delta+\frac{\alpha d}{2}\left(\frac{1}{q}-\frac{1}{p}\right)}\left\|u_{0}\right\|_{L^{q}\left(\mathbb{R}^{d}\right)},
\end{aligned}
$$

the last inequality can be obtained by using the conditions $\delta>\frac{\alpha d(q-p)}{2 p q}$ and $\mu>0$. From this result, we can conclude that $E_{\alpha, 1}\left[\Delta(I-k \Delta)^{-1} t^{\alpha}\right] u_{0} \in C_{\delta, \mu}\left((0, T] ; Z_{0}\right)$.

Next, we have to show that $\mathscr{K}$ is a contraction mapping on $C_{\delta, \mu}\left((0, T] ; Z_{0}\right)$. Indeed, let $v_{1}, v_{2} \in C_{\delta, \mu}\left((0, T] ; Z_{0}\right)$, combining (4.8), (4.9), and Lemma 4.1 yields that

$$
\begin{aligned}
\| & (I-k \Delta)^{-1} \int_{0}^{t}(t-m)^{\alpha-1} E_{\alpha, \alpha}\left[\frac{\Delta(I-k \Delta)^{-1}}{(t-m)^{1-\alpha}}\right]\left[F\left(v_{1}(m)\right)-F\left(v_{2}(m)\right)\right] \mathrm{d} m \|_{Z_{0}} \\
\leq & \int_{0}^{t}(t-m)^{\alpha-1}\left\|E_{\alpha, \alpha}\left[\frac{\Delta(I-k \Delta)^{-1}}{(t-m)^{1-\alpha}}\right](I-k \Delta)^{-1}\left[F\left(v_{1}(m)\right)-F\left(v_{2}(m)\right)\right]\right\|_{Z_{0}} \mathrm{~d} m \\
\leq & C \int_{0}^{t}(t-m)^{\alpha-1}\left\|(I-k \Delta)^{-1}\left[F\left(v_{1}(m)\right)-F\left(v_{2}(m)\right)\right]\right\|_{L^{p}\left(\mathbb{R}^{d}\right)} \mathrm{d} m \\
& +C \int_{0}^{t}(t-m)^{\alpha\left[1+\frac{d}{2}\left(\frac{1}{q}-\frac{1}{p}\right)\right]-1}\left\|(I-k \Delta)^{-1}\left[F\left(v_{1}(m)\right)-F\left(v_{2}(m)\right)\right]\right\|_{Z_{0}} \mathrm{~d} m . \\
& :=C\left(I_{1}+I_{2}\right) .
\end{aligned}
$$

Thanks to Lemma 4.1 and the condition (2.15), we can estimate $I_{1}, I_{2}$ as

$$
\begin{aligned}
t^{\delta} e^{-\mu t} I_{1} & \leq \widetilde{C} C_{F} t^{\delta} \int_{0}^{t} e^{-\mu(t-m)}(t-m)^{\alpha-1} m^{-\delta}\left(m^{\delta} e^{-\mu m}\left\|v_{1}(m)-v_{2}(m)\right\|_{L^{p}\left(\mathbb{R}^{d}\right)}\right) \mathrm{d} m \\
& \leq \widetilde{C} C_{F}\left(t^{\delta} \int_{0}^{t} e^{-\mu(t-m)}(t-m)^{\alpha-1} m^{-\delta} \mathrm{d} m\right)\left\|v_{1}-v_{2}\right\|_{C_{\delta, \mu}\left((0, T] ; Z_{0}\right)} .
\end{aligned}
$$

Analogously, the following holds

$$
t^{\delta} e^{-\mu t} I_{2} \leq \widetilde{C} C_{F}\left(t^{\delta} \int_{0}^{t} e^{-\mu(t-m)}(t-m)^{\alpha\left[1+\frac{d}{2}\left(\frac{1}{q}-\frac{1}{p}\right)\right]-1} m^{-\delta} \mathrm{d} m\right)\left\|v_{1}-v_{2}\right\|_{C_{\delta, \mu}\left((0, T] ; Z_{0}\right)} .
$$

From the two above estimates, we obtain

$$
\begin{aligned}
& \left\|(I-k \Delta)^{-1} \int_{0}^{t}(t-m)^{\alpha-1} E_{\alpha, \alpha}\left[\frac{\Delta(I-k \Delta)^{-1}}{(t-m)^{1-\alpha}}\right]\left[F\left(v_{1}(m)\right)-F\left(v_{2}(m)\right)\right] \mathrm{d} m\right\|_{C_{\delta, \mu}\left((0, T] ; Z_{0}\right)} \\
& \leq C\left[\mathcal{Q}_{1}(\mu)+\mathcal{Q}_{2}(\mu)\right]\left\|v_{1}-v_{2}\right\|_{C_{\delta, \mu}\left((0, T] ; Z_{0}\right)},
\end{aligned}
$$


where we set

$$
\left\{\begin{array}{l}
\mathcal{Q}_{1}(\mu):=t^{\delta} \int_{0}^{t} m^{-\delta} e^{-\mu(t-m)}(t-m)^{\alpha-1} \mathrm{~d} m \\
\mathcal{Q}_{2}(\mu):=t^{\delta} \int_{0}^{t} m^{-\delta} e^{-\mu(t-m)}(t-m)^{\alpha\left[1+\frac{d}{2}\left(\frac{1}{q}-\frac{1}{p}\right)\right]-1} \mathrm{~d} m
\end{array}\right.
$$

By using (2.16) and the same arguments as the above, yields that

$$
\begin{aligned}
& \left\|\int_{0}^{t} \int_{0}^{m} \frac{(I-k \Delta)^{-1}}{(t-m)^{1-\alpha}} E_{\alpha, \alpha}\left[\frac{\Delta(I-k \Delta)^{-1}}{(t-m)^{1-\alpha}}\right]\left[h\left(m-s, v_{1}(m)\right)-h\left(m-s, v_{2}(m)\right)\right] \mathrm{d} s \mathrm{~d} m\right\|_{C_{\delta, \mu}\left((0, T] ; Z_{0}\right)} \\
& \leq C\left[\mathcal{Q}_{3}(\mu)+\mathcal{Q}_{4}(\mu)\right]\left\|v_{1}-v_{2}\right\|_{C_{\delta, \mu}\left((0, T] ; Z_{0}\right)},
\end{aligned}
$$

where we set

$$
\left\{\begin{array}{l}
\mathcal{Q}_{3}(\mu):=t^{\delta} \int_{0}^{t} m^{-\delta} e^{-\mu(t-m)}(t-m)^{\alpha-1}\left(m^{\delta} \int_{0}^{m}(m-s)^{-\beta} s^{-\delta} e^{-\mu(m-s)} \mathrm{d} s\right) \mathrm{d} m \\
\mathcal{Q}_{4}(\mu):=t^{\delta} \int_{0}^{t} m^{-\delta} e^{-\mu(t-m)}(t-m)^{\alpha\left[1+\frac{d}{2}\left(\frac{1}{q}-\frac{1}{p}\right)\right]-1}\left(m^{\delta} \int_{0}^{m}(m-s)^{-\beta} s^{-\delta} e^{-\mu(m-s)} \mathrm{d} s\right) \mathrm{d} m .
\end{array}\right.
$$

On account of (4.15) and (4.16), we have

$$
\left\|\mathscr{K} v_{1}-\mathscr{K} v_{2}\right\|_{C_{\delta, \mu}\left((0, T] ; Z_{0}\right)} \leq C\left(\sum_{j=1}^{4} \mathcal{Q}_{j}\right)\left\|v_{1}-v_{2}\right\|_{C_{\delta, \mu}\left((0, T] ; Z_{0}\right)} .
$$

It follows from the conditions of $p, q, \delta$ we have established that

$$
\alpha+\frac{\alpha d}{2}\left(\frac{1}{q}-\frac{1}{p}\right)>0, \quad-\delta>-1, \quad \delta+\alpha+\frac{\alpha d}{2}\left(\frac{1}{q}-\frac{1}{p}\right)>0, \quad-\delta+\alpha>0, \quad \delta+\beta<1 .
$$

By applying Lemma 3.2, the following limits hold

$$
\begin{aligned}
- & \lim _{\mu \rightarrow \infty}\left(\sup _{0 \leq t \leq T} t^{\delta} \int_{0}^{t} m^{-\delta} e^{-\mu(t-m)}(t-m)^{-\beta} \mathrm{d} m\right) \\
= & \lim _{\mu \rightarrow \infty}\left(\sup _{0 \leq t \leq T} t^{1-\beta} \int_{0}^{1} m^{-\delta} e^{-\mu t(1-m)}(1-m)^{-\beta} \mathrm{d} m\right)=0
\end{aligned}
$$

- $\lim _{\mu \rightarrow \infty}\left(\sup _{0 \leq t \leq T} t^{\delta} \int_{0}^{t} m^{-\delta} e^{-\mu(t-m)}(t-m)^{\alpha-1} \mathrm{~d} m\right)$

$$
=\lim _{\mu \rightarrow \infty}\left(\sup _{0 \leq t \leq T} t^{\alpha} \int_{0}^{1} m^{-\delta} e^{-\mu t(1-m)}(1-m)^{\alpha-1} \mathrm{~d} m\right)=0
$$

- $\lim _{\mu \rightarrow \infty}\left(\sup _{0 \leq t \leq T} t^{\delta} \int_{0}^{t} m^{-\delta} e^{-\mu(t-m)}(t-m)^{\alpha\left[1+\frac{d}{2}\left(\frac{1}{q}-\frac{1}{p}\right)\right]-1} \mathrm{~d} m\right)$

$$
=\lim _{\mu \rightarrow \infty}\left(\sup _{0 \leq t \leq T} t^{\alpha\left[1+\frac{d}{2}\left(\frac{1}{q}-\frac{1}{p}\right)\right]} \int_{0}^{1} m^{-\delta} e^{-\mu t(1-m)}(1-m)^{\alpha\left[1+\frac{d}{2}\left(\frac{1}{q}-\frac{1}{p}\right)\right]-1} \mathrm{~d} m\right)=0 .
$$


Hence, we can find a positive number $\mu^{*}$ that is large enough to ensure the existence of a constant $\mathcal{L}<1$ such that

$$
\left\|\mathscr{K} v_{1}-\mathscr{K} v_{2}\right\|_{C_{\delta, \mu}\left((0, T] ; Z_{0}\right)} \leq \mathcal{L}\left\|v_{1}-v_{2}\right\|_{C_{\delta, \mu}\left((0, T] ; Z_{0}\right)}
$$

Which proves that $\mathscr{K}$ is a contraction mapping on $C_{\delta, \mu}\left((0, T] ; Z_{0}\right)$. We complete the proof by using Banach fixed point theorem to conclude that problem (1.1) has a unique mild solution $u$ in $C_{\delta, \mu}\left((0, T] ; Z_{0}\right)$.

\subsection{Application}

In view of the theory result, we consider the following initial value problem

$$
\begin{cases}{ }_{C} D_{t}^{1 / 2}(u-k \Delta u)=\Delta u+\lambda_{1} u+\frac{1}{\Gamma(1-\beta)} \int_{0}^{t} e^{(z-t)}(t-z)^{-1 / 2} u(z) \mathrm{d} z, & \text { in } \mathbb{R}^{4} \times(0, T), \\ u=\mathcal{A}>0 & \text { in } \mathbb{R}^{4} \times\{0\} .\end{cases}
$$

By choosing $\delta=0,3$ and a sufficiently large $\mu=\mu_{0}$, Theorem 4.2 can be applied to ensure the existence and uniqueness of the mild solution in the space $C_{3 / 10, \mu_{0}}\left((0, T] ; L^{5}\left(\mathbb{R}^{4}\right) \cap L^{20}\left(\mathbb{R}^{4}\right)\right)$.

\section{Conclusion}

In this study, we derive the global existence and uniqueness for the mild solution of the initial value problem associated with the time-fractional pseudo-parabolic equations with globally Lipschitz source terms. In general, we provide the theory results and examples for problem (1.1) on $\mathbb{R}^{N}$ as well as a smooth bounded domain $\mathcal{D}$ of $\mathbb{R}^{N}$.

Acknowledgements. This research is supported by Industrial University of Ho Chi Minh City (IUH) under grant number 66/HD-DHCN.

\section{REFERENCES}

[1] B. Andrade and A. Viana, Abstract Volterra integrodifferential equations with applications to parabolic models with memory. Math. Ann. 369 (2017) 1131-1175.

[2] J.M. Arrieta and A.N. Carvalho, Abstract parabolic problems with critical nonlinearities and applications to Navier-Stokes and heat equations. Trans. Am. Math. Soc. 352 (2000) 285-310.

[3] D. Baleanu, B. Shiri, H.M. Srivastava and M. Al Qurashi, A Chebyshev spectral method based on operational matrix for fractional differential equations involving non-singular Mittag-Leffler kernel. Adv. Diff. Equ. 2018 (2018) 353.

[4] D. Baleanu, A. Jajarmi, H. Mohammadi and S. Rezapour, A new study on the mathematical modelling of human liver with Caputo-Fabrizio fractional derivative. Chaos Solitons Fract. 134 (2020) 109705.

[5] D. Baleanu, A. Jajarmi, S.S. Sajjadi and J.H. Asad, The fractional features of a harmonic oscillator with position-dependent mass. Commun. Theor. Phys. 72 (2020) 055002-55010.

[6] M.Kh. Beshtokov, Boundary value problems for a pseudo-parabolic equation with the Caputo fractional derivative. Differ. Equ. 55 (2019) 884-893.

[7] Y. Cao, J. Yin and C. Wang, Cauchy problems of semilinear pseudo-parabolic equations. J. Diff. Equ. 246 (2009) $4568-4590$.

[8] Y. Cao, J. Yin and C. Wang, Cauchy problems of semilinear pseudo-parabolic equations. J. Diff. Equ. 246 (2009) $4568-4590$.

[9] Y. Chen, H. Gao, M. Garrido-Atienza and B. Schmalfuß, Pathwise solutions of SPDEs driven by Hölder-continuous integrators with exponent larger than $1 / 2$ and random dynamical systems. Disc. Cont. Dyn. Syst. Ser. A 34 (2014) $79-98$.

[10] W. Chen and C. Li, Maximum principles for the fractional $p$-Laplace and symmetry of solutions. Adv. Math. 335 (2018) $735-758$.

[11] H. Chen and S. Tian, Initial boundary value problem for a class of semilinear pseudo-parabolic equations with logarithmic nonlinearity. J. Differ. Equ.. 258 (2015) 4424-4442.

[12] E. Di Nezza, G. Palatucci and E. Valdinoci, Hitchhiker's guide to the fractional Sobolev spaces. Bull. Sci. Math. 136 (2012) $521-573$.

[13] R. Gorenfloo, A.A. Kilbas and F. Mainardi, Mittag-Leffler Functions, Related Topics and Applications. Springer, Berlin (2014).

[14] A. Jajarmi, A. Yusuf, D. Baleanu and M. Inc, A new fractional HRSV model and its optimal control: a non-singular operator approach. Physica A 547 (2020) 123860. 
[15] A Jajarmi and D. Baleanu, On the fractional optimal control problems with a general derivative operator. Asian J. Cont. (2019). https://doi.org/10.1002/asjc.2282

[16] S. Ji, J. Yin and Y. Cao, Instability of positive periodic solutions for semilinear pseudo-parabolic equations with logarithmic nonlinearity. J. Differ. Equ. 261 (2016) 5446-5464.

[17] L. Jin, L. Li and S. Fang, The global existence and time-decay for the solutions of the fractional pseudo-parabolic equation. Comput. Math. Appl. 73 (2017) 2221-2232.

[18] E.D. Khiabani, H. Ghaffarzadeh, B. Shiri and J. Katebi, Spline collocation methods for seismic analysis of multiple degree of freedom systems with visco-elastic dampers using fractional models. J. Vibr. Contr. 26 (2020) 1445-1462.

[19] W. Lian, J. Wang and R. Xu, Global existence and blow up of solutions for pseudo-parabolic equation with singular potential. J. Diff. Equ. 269 (2020) 4914-4959.

[20] Y. Liu, W. Jiang and F. Huang, Asymptotic behaviour of solutions to some pseudo-parabolic equations. Appl. Math. Lett. 25 (2012) 111-114.

[21] F. Mainardi, A. Mura and G. Pagnini, The M-Wright function in time-fractional diffusion processes: a tutorial survey. Int. J. Differ. Equ. 2010 (2010) Art. ID 104505

[22] B.B. Mandelbrot and J.W.V Ness, Fractional Brownian motions, fractional noises and applications. SIAM Rev. 10 (1968) 422-437.

[23] V. Padron, Effect of aggregation on population recovery modeled by a forward-backward pseudo-parabolic equation. Trans. Amer. Math. Soc. 356 (2004) 2739-2756.

[24] J.E.M. Rivera and L.H. Fatori, Smoothing effect and propagations of singularities for viscoelastic plates. J. Math. Anal. Appl. 206 (1997) 397-427.

[25] M.K. Sadabad, A.J. Akbarfam and B. Shiri, A numerical study of eigenvalues and eigenfunctions of fractional Sturm-Liouville problems via Laplace transform. Indian J. Pure Appl. Math. 51 (2020) 857-868.

[26] S.S. Sajjadi, D. Baleanu, A. Jajarmi and H.M. Pirouz, A new adaptive synchronization and hyperchaos control of a biological snap oscillator. Chaos Solitons Fract. 138 (2020) 109919, 13 pp.

[27] B. Shiri, G.-C. Wu and D. Baleanu, Collocation methods for terminal value problems of tempered fractional differential equations. Appl. Numer. Math. 156 (2020) 385-395.

[28] B. Shiri and D. Baleanu, Numerical solution of some fractional dynamical systems in medicine involving non-singular kernel with vector order. Results Nonlinear Anal. 2 (2019) 160-168.

[29] B. Shiri and D. Baleanu, System of fractional differential algebraic equations with applications. Chaos Solitons Fractals. 120 (2019) 203-212.

[30] R.E. Showalter and T.W. Ting, Pseudo-parabolic partial differential equations. SIAM J. Math. Anal. 1 (1970) 1-26.

[31] N.H. Tuan, V.V. Au and R. Xu, Semilinear Caputo time-fractional pseudo-parabolic equations. Commun. Pure Appl. Anal. 20 (2021) 583-621.

[32] R. Xu and J. Su, Global existence and finite time blow-up for a class of semilinear pseudo-parabolic equations. J. Funct. Anal. 264 (2013) 2732-2763. 